\title{
ORGAN DONATION: AN EDUCATIONAL PACK FOR HIGH SCHOOL STUDENTS
}

\author{
Styliani Kapsali, Vasiliki Sapountzi, Alexandra Nestora, \& Lefkothea-Vasiliki Andreou \\ Department of Biological Applications and Technologies, University of Ioannina (Greece)
}

\begin{abstract}
Blood, bone marrow and organ donation is a major issue that concerns all of us. Even though 18 is the age of eligibility to become a donor, awareness should be raised earlier through education. To this end, and given that this issue is marred by prejudice and misconceptions, we developed an educational pack, aimed at senior high school students, which may be delivered in the context of a biology or sociology course. The proposed teaching intervention follows a three-fold approach: (1) First, students are educated on monoclonal antibodies and relevant biotechnology tools and their role in organ transplantation via a board game, (2) Second, we promote empathy using a free writing exercise that employs art prompts and audiovisual material, (3) Third, we assign students with research projects that involve building questionnaires, collecting and analyzing data and producing a science poster to be posted on the school website. Importantly, the aim of this proposed intervention is to educate and to raise awareness while students build on their research, technology and writing skills.
\end{abstract}

Keywords: Organ donation, science literacy, research thinking, active learning, secondary education.

\section{Introduction}

As the famous quote of Bonnie Arbon goes, "You are a piece of the puzzle of someone else's life. You may never know where you fit, but others will fill the holes in their lives with pieces of you." (https://helphopelive.org/). Recent studies have shown that there is a rise in organ donation demand. More specifically, in the United States of America a new patient will need a transplant every ten minutes (Bastani, 2020), as opposed to every fifteen minutes ten years ago (Abouna, 2008). It is therefore crucial that this issue is effectively communicated as it involves awareness, empathy, and social cohesion, thus reflecting social culture (Parker et al., 2009). Nevertheless, controversy and misconceptions surround organ donation (Morgan et al., 2008). In the present micro-scenario, we aim to promote awareness in an interdisciplinary educational approach.

\section{Methodology}

Subject: Biology, Sociology - Topic: Organ donation - Age group: high school students and young adults - Time: 90 minutes.

\subsection{Educational goals}

Through this educational pack we aim to: (1) Educate students on biological and biotechnological topics relevant to organ donation, (2) Introduce students to blood, bone marrow and organ donation procedures, (3) Introduce students to pre-writing strategies, (4) Alleviate students' writing apprehension, (5) Encourage artistic expression, (6) Introduce students to research thinking and methodology, (7) Improve students' research and collaboration skills, (8) Raise awareness on health issues, (9) Promote empathy and highlight the social benefits of volunteering, (10) Familiarize students with complicated and sensitive science and social issues, (11) Promote science, technology and visual literacy, (12) Practice interdisciplinary (i.e., Biology, Sociology, Art, Research), critical thinking and active learning educational approaches. 


\subsection{Materials}

(1) IT room with Internet access, (2) Laptop, (3) Projector, (4) Speakers, (5) A4 paper sheets, (6) Printer, (7) Printing material for the board game activity.

\subsection{Procedure}

The present micro-scenario has been developed to educate and raise awareness on the topic of blood, bone marrow and organ donation. The teaching intervention spans over two teaching hours. In the first phase, we discuss science topics related to donation by means of an educational board game. During the second phase, the students engage in art and research activities. We conclude with self-reflection and an evaluation of the proposed lesson.

2.3.1. 1st Phase (time: 45 min). 1st micro-activity (time: $45 \mathrm{~min}$ ). Introduction to biology and biotechnology topics concerning organ donation using an educational board game activity.

1. Warmer: We project Sir Robert Lechler's TEDxLondonBusinessSchool talk entitled "The future of Organ Donation" (https://www.ted.com/talks/robert_lechler_the_future_of_organ_transplants). We prompt students to discuss their feelings and thoughts on the talk transcript.

2. Next, students are divided into groups of 3-5 people. Students are randomly assigned to groups with the help of a Random Sequence Generator algorithm (https://www.random.org/sequences/).

3. We explain to students that they will engage in an educational board game activity, namely "Make life last!". The game pertains the following topics: monoclonal antibodies, the antigen-antibody relationship, organ/tissue preservation, organ, bone marrow and blood donation \& 3D organ printers/nanobiotechnology.

4. We hand to each group the following: a packet of double-sided cards, an answer sheet, a pack of double-sided colored badges.

5. We explain the game rules to the class: once a group has written down the answer to a card, the team can move their respective question badge on the gameboard. The team that first reaches the finish line having answered all questions is the winning team. The winning team can choose the destination of the next field trip, decide who the next guest speaker of the class will be or pick an organization of their preference for the class to visit.

Note to the instructor: The cards have been designed using a Google Docs Slides template (https://www.google.com/slides/about/) and are loosely based and inspired by Science Museum "Who am I?" flashcards (https://learning.sciencemuseumgroup.org.uk/resources/talking-points-cards/). The game materials can be found online at the "Make life last!" designated Google Folder (https://drive.google.com/drive/folders/1JEVjGSPQAmImPxUZTrsJ8jjHYMKJE1uG?usp=sharing). The teacher should print out the cards in advance of the lesson as well as the answer sheets and the badges. The gameboard can be designed to the teacher's preference using an online application such as "Board Games Maker" (https://www.boardgamesmaker.com/) or "The Game Crafter" (https://www.thegamecrafter.com/). Alternatively, the board can be hand-crafted, or a line-up of colored boxes may be used instead.

2.3.2. 2nd Phase (duration: 45 minutes). 2nd micro-activity (duration: 20 minutes). Empathy on the issue of organ donation is promoted by means of a free writing exercise that involves art prompts. Students are thus introduced to pre-writing strategies and artistic inspiration is employed to alleviate writing apprehension, i.e., writing-related stress.

1. A discussion is held on students' feelings and attitudes towards writing. We explain that stress-related writing, namely writer's block, is a common problem that may be alleviated through pre-writing strategies, such as free writing (Andreou et al., 2018).

2. Students are handed out blank, white sheets of paper. We explain to them that they will engage in a free writing activity where a series of art prompts, pertinent to the topic of organ donation, will be projected. Following each prompt, they will be asked to write freely and non-stop, without planning or attention to structure or editing.

3. First, an excerpt of the video "Transplant" (03:42 - 06:42), of the art project by the same name (http://www.transplantproject.com/video), by artists Tim Waiwright \& John Wynne is projected. Following the video, and in response to it, the students are instructed to write freely and non-stop for 3 min. Trigger warning: the video contains a still of a bloody cloth and a sound with a high pitch.

4. Second, the video art "Tunnel" (https://www.vasileiadereli.com/copy-of-solo) by artist Vasileia Dereli is projected (duration 02:53). Students are again requested to write for the next three minutes. Trigger warning: contains an agonizing scene of skin stretching that some may find disturbing.

5. Finally, the painting "A French underground hospital at Verdun" (https://wellcomecollection.org/works/nuy2gd3t) by Ugo Matania is projected while the music piece "The 
firebird" by Igor Stravinsky is reproduced (duration 03:30) (https://www.youtube.com/watch?v=wWQa8ZdAeIM\&ab_channel=LondonSymphonyOrchestra-Topic). Students are again requested to write.

2.3.3. 2nd Phase (duration: 45 minutes). 3rd micro-activity (duration: 25 minutes). Introduction of students to research thinking and methodology via a science poster activity. Students collect data concerning organ donation and communicate their findings.

1. We instruct students to run a Google Scholar search (https://scholar.google.com/) using organ transplantation as a search term. We ask them to study the results returned and explain to them that the site is a repository of reliable science and research publications.

2. Students are instructed to work in groups and come up with ten questions exploring the social aspects of organ donation, e.g., common misconceptions. We then ask them to build their questionnaires on Google Forms (https://www.google.com/forms). The questionnaires will be later posted on the school website so that data for a sociological study may be collected.

Homework project \#1: Students are handed-in instructions on how to run a basic descriptive statistics analysis on their data. Note for the instructor: This activity could be a joint project with their Math instructor. With regards to data analysis, useful instructions may be drawn by the dummies.com website (https://www.dummies.com/software/microsoft-office/excel/how-to-use-excels-descriptivestatistics-tool/).

Homework project \#2: Students are requested to build a science poster on Canva (https://www.canva.com/el_gr/) so to effectively present and communicate their research findings. For their posters, we instruct them to use copyright-free images from repositories such as the Wikimedia Commons (https://www.wikimedia.org/).

3. We ask students to self-reflect on the learning outcomes of their engagement with the activities described in the present micro-scenario. We then hold a plenary discussion on the educational intervention.

\section{Discussion}

Organ donation is a topic somehow overlooked in education; however, it has been shown that when part of the curriculum it promotes understanding and health literacy (Siebelink et al., 2017). This may be due to the interdisciplinary, complicated, and sensitive nature of the issue. Also, creating a safe space in class (Holley \& Steiner, 2005) is substantial for the topic to be discussed and analyzed. In the present micro-scenario, we propose an educational pack for high school students. The relevant science topics are communicated via an educational board game that we have constructed for the purposes of this project. Empathy is raised by means of art and writing. Finally, the social aspects of organ donation are investigated though research posters. The effectiveness of the proposed educational pack should be further investigated in terms of the learning outcomes and the student experience.

\section{References}

Abouna, G. M. (2008). Organ shortage crisis: problems and possible solutions. Transplantation Proceedings, 40, 34-38.

Andreou, L.-V., Aletra, V., Athanasopoulou, G., \& Psarropoulou, C. (2018). Good practices in teaching English for scientific purposes to Biology students in higher education. INTED2018 Proceedings, 7174-7183.

Bastani, B. (2020). The present and future of transplant organ shortage: some potential remedies. Journal of Nephrology, 33, 277-288.

Holley, L., \& Steiner, S. (2005). Safe space: student perspectives on classroom environment. Journal of Social Work Education, 41, 49-64.

Morgan, S. E., Harrison, T. R., Afifi, W. A., Long, S. D., \& Stephenson, M. T. (2008). In their own words: the reasons why people will (not) sign an organ donor card. Health Communication, 23, 23-33.

Parker, J., Saklofske, D., Wood, L., \& Collin, T. (2009). The role of emotional intelligence in education. In Assessing emotional intelligence (pp. 239-255). Boston, MA: Springer.

Siebelink, M., Verhagen, A., Roodbol, P., Albers, M., \& Van de Wiel, H. (2017). Education on organ donation and transplantation in primary school; teachers' support and the first results of a teaching module. Plos One, 12. 